\title{
Analysis on the Current Situation of Classified Management of Drug Shortages: Case China
}

\section{Bing Fang, Hui Chen, Xiaohe Li, Jianhong Yang and Zhen Chen*}

School of Pharmaceutical Sciences, Zhengzhou University, China

*Corresponding Author: Zhen Chen, School of Pharmaceutical Sciences, Zhengzhou University, China.
Received: March 04, 2021

Published: March 22, 2021

(C) All rights are reserved by Zhen Chen., et al.

\begin{abstract}
Drug shortage is a worldwide problem, it will not only have a serious impact on the treatment of patients, but also increase the work and financial burden of the health care system and health care providers. Various countries and regions have established different prevention and mitigation measures.

China's central and local governments have introduced a series of measures to strengthen the management of drug shortage, but the effect is not ideal. Due to the different nature of the shortage of drugs, the management methods should be different, so we should comprehensively consider the various attributes of the shortage of drugs, establish a classification method to manage the shortage of drugs, so as to improve the efficiency of the existing measures, and supplement and improve the inadequate. There are some limitations in the current classification management system of shortage drugs in China. The classification index cannot comprehensively summarize the attributes of shortage drugs, and the judgment standard of each index is fuzzy. Based on the current situation of shortage drugs in China and the existing research experience at home and abroad, this paper puts forward that the elements of classification management of shortage drugs in China are clinical importance, the availability of alternative drugs and the impact of shortage, and describes the judgment criteria of each index in detail.
\end{abstract}

Keywords: Drug Product Shortage; Substitutable Therapy; Policy Advice; Comparative Study

\section{Introduction}

Drug shortages is still a global problem, which has a serious impact on health care personnel, medical institutions, patients and regulatory agencies in various countries [1-3]. Especially during the COVID-19 pandemic, it will not only affect the supply of most drugs and make the problem of drug shortage more prominent, but also cause supply and demand problems of some drugs that can alleviate the symptoms caused by COVID-19, for example, paracetamol, etc. In addition, there are additional potential impacts on patient care $[4,5]$. In order to ensure the public access to drugs, China's regulatory authorities have always attached great impor- tance to the problem of drug shortages, and comprehensively used a variety of measures to solve the problem of drug shortages. For example, in order to ensure the production and supply of drugs in short supply, fixed-point production, centralized production, normalized storage and other methods have been adopted; in order to ensure the demand for clinical treatment, China's regulatory authorities have successively issued a series of guidelines and documents to provide technical guidance for effective management of drugs in short supply and scientific selection of alternative drugs for medical institutions. Although the measures and means to solve the shortage problem have been constantly enriched and impro- 
ved, they have not fully played their due role. This is mainly due to the fact that the shortage of drugs involves a variety of drugs in different treatment fields. Not only the clinical importance of drugs, but also the degree of impact on the public after the shortage, the corresponding measures and measures should be different. China's regulatory authorities issued a guideline in 2019, which requires medical institutions to classify and manage the drugs in shortage according to the clinical necessity of drugs in shortage and the availability of alternative drugs [6]. However, the guideline is only from the perspective of medical institutions and cannot be used as a general classification standard. Therefore, we should establish a more comprehensive classification method to evaluate the drug attributes, so as to establish the priority of shortage drugs and formulate different measures for different shortage drugs, so as to make the control strategy of shortage drugs more flexible and play a greater efficiency.

\section{Materials and Methods}

Current situation of classified management of drug shortages in China

Before that, the central government and some local governments have established a simple classification method of drugs in short supply. In 2019, the Chinese Health Commission issued a classification method for shortage drugs, which divided the shortage drugs into two categories: clinically necessary and irreplaceable, clinically necessary and replaceable (or not completely replaceable). According to the expected time of inventory and supply recovery, the shortage degree is divided into three levels: (1) level 1 shortage, which means that the supply cannot be normal for six consecutive months or more; (2) Level 2 shortage, which means that the supply cannot be normal for three consecutive months or more; (3) Level 3 shortage, which means that the supply cannot be normal for one consecutive month or more. If the supply cannot be expected to resume, it will be treated as the first level shortage [6]. On the one hand, in this classification method, there are some ambiguities in using the combination of clinical necessity and the availability of alternative drugs as a measure to describe the clinical importance of drugs. "Clinical necessity" already contains the meaning that there is no suitable alternative drugs or alternative drugs cannot be provided, so the classification of shortage drugs is not accurate enough. From the perspective of medical institutions, this classification method takes the clinical importance of shortage drugs and the availability of alternative drugs as the main classification indicators, which is worthy of recognition. However, it only considers the shortage drugs with important clinical value and ignores the potential value of the shortage drugs with insignificant clinical value, and does not comprehensively classify all clinical drugs. On the other hand, through the investigation and study of 26 medical institutions in China, the researchers found that the average shortage time of drugs was 15.83 months, which indicated that when the shortage time was used as the grading index, the grading judgment standard was not accurate, and the measurement selection should be modified and corrected [7]. Although the regulatory authorities have initially put forward the classification method, it will produce large errors in the classification of drugs in short supply, which needs to be further optimized and improved. According to the drug supply situation in Shandong Province, the shortage drugs are divided into three categories: the first category is shortage drugs: out of stock; the second category is shortage drugs: under normal demand, the supply is insufficient or not in time, and the monthly arrival rate is less than $60 \%$; the third category is shortage drugs: under the known shortage situation, a small number of orders, a small amount of supply [8]. Obviously, this classification method takes the market supply and demand situation of the shortage drugs as the index and ignores the important attribute of the life relevance of drugs, so it cannot be used as the classification standard to comprehensively summarize the characteristics of the shortage drugs. In addition, if only the market attribute of drugs in short supply is used as the main index to classify and formulate different mitigation strategies, some drug manufacturers may manipulate the market to obtain improper benefits. Some medical institutions in China introduce $\mathrm{ABC}$ classification in the process of managing the shortage of drugs, and divide the drugs into $\mathrm{ABC}$ three categories: Class $A$ is the drugs with high price and large dosage, class $C$ is the drugs with low price and small dosage, and class $B$ is the varieties between $A$ and $C[9,10]$. This kind of classification method is used as a tool to strengthen the inventory management of medical institutions, to a certain extent, to avoid drug shortages and ensure clinical medication. However, this classification method only discusses the price and clinical dosage of drugs and cannot accurately describe the characteristics of drugs in shortage, and the classification standard is vague, which does not have the reference significance to establish the classification method of drugs in shortage. 
Study on the classification of drug shortages

In order to continuously improve the prevention and response mechanism and measures of drug shortages and better guarantee the drug supply, some experts or scholars in many countries and regions have studied the classified management of drug shortages. Sheng divided the drugs into four levels according to the degree of emergency, the degree of harm, the scope of involvement and other indicators.

Level 1: clinical emergency (rescue) drugs; Level 2: drugs for the treatment of major diseases; Level 3: clinically necessary and irreplaceable general drugs; level 4: clinically necessary but replaceable drugs [11]. According to the shortage of different levels, different measures should be taken. In this classification method, clinical medicine includes emergency medicine and medicine for serious diseases in a certain sense, so it is easy to misunderstand the classification of shortage medicine. In fact, when most experts and scholars study the problem of drug shortages, they generally use anatomical therapeutics and chemical classification system ATC (Anatomical Therapeutic Chemical, ATC) is a simple classification of drugs. As an official classification system, this classification method is only for the convenience of studying or explaining problems, and cannot reflect the impact of shortage of drugs on patients and other characteristics, which has great limitations. The Parenteral DrugAssociation (PDA) classifies the importance of drugs in shortage according to the therapeutic use/indication of drugs, the clinical needs of patients, the possibility of shortage and the availability of alternative drugs. These classification bases comprehensively consider the property of drugs, the impact on patients, alternative drugs, etc., which is a more comprehensive classification method [12]. Similarly, considering the EU as a special group, Umberto M. Musazzi and others proposed a classification model based on the therapeutic use of drugs in short supply, the availability of alternative drugs and the market share of products. The model can be flexibly applied according to the different needs of Member States [13]. FDA focuses on the management of the shortage of medically essential drugs. Medically essential drugs are used to treat or prevent serious diseases or medical conditions, and the medical staff think that there is no sufficient supply of substitutes. Although FDA focuses on the management of medically essential drugs, it will not ignore the shortage of other drugs in order to prevent possible public health impacts [14]. This division greatly improves the efficiency of management and control, and makes the limited measures play a greater role. EMA classifies the shortage drugs into key drugs and other drugs according to the therapeutic use and the availability of alternative drugs. It should be pointed out that when there are alternative manufacturing sites for the same product, generic drugs and different strength/dosage forms of the same product, they will not be defined as key drugs [15].

Based on the above analysis, there are two important aspects in the process of establishing the classification system of shortage drugs. On the one hand, it is necessary to select and determine the appropriate classification index; on the other hand, it is necessary to describe the classification basis and judgment standard in detail to improve the practicability and accuracy of classification.

\section{Results and Discussion}

\section{Selection of classification index of drug shortages}

Chinese scholars have found that clinical use, regulatory authorities and production are the key factors affecting and evaluating the shortage of essential drugs [16]. The impact of drug shortages on the treatment process of patients should be placed in the first place. Once there is a shortage of key drugs with important clinical treatment value, the serious consequences will be greater than those of other drugs. For example, when there is a shortage of drugs for the treatment of cancer, it will not only affect the treatment process of patients, but also produce other risk factors, and even endanger the lives of patients. Therefore, most of foreign regulatory agencies take this as one of the main criteria for the classification of drugs in short supply. Secondly, when there is a shortage of drugs, the preferred mitigation strategy of medical institutions is to choose appropriate alternative drugs to ensure the treatment of patients, and the use of alternative drugs is the only feasible medium and long-term strategy. A study of medical institutions in the United States shows that $84.3 \%$ of the respondents will adopt the mitigation strategy of using alternative drugs when there is a shortage of drugs [17]. If an inappropriate alternative drug is selected, it may affect the treatment process of patients because the clinical efficacy of alternative drugs is not significant or increase the cost of treatment or may lead to patients having to give up treatment. Therefore, most of the classification systems in foreign policies, reports and literatures take the availability of alternative drugs as one of the indicators. Based on the above considerations, we continue to use the clinical importance and the availability of alternative drugs as the classification indicators of shortage drugs, and recombine these two key indicators, and divide the shortage drugs into 
four categories: clinically important and irreplaceable, clinically important and replaceable (complete or partial substitution), clinically unimportant and irreplaceable, and clinically unimportant and replaceable Generation (complete or partial replacement). It is worth noting that only considering the impact on patients (clinical importance, availability of alternative drugs) when establishing the classification system is not enough to fully describe all the attributes of shortage drugs. In addition to the clinical importance and the availability of alternative drugs, we should also pay more attention to the impact of drug shortages (such as the expected supply recovery time, national market share, etc.). Comprehensive evaluation can improve the efficiency of relevant means or measures, avoid the waste of administrative resources.

\section{Classification index judgment standard}

According to the therapeutic use of drugs, the clinical importance is defined as: (a) for the diagnosis and treatment of life-threatening or seriously damaging the quality of life; and (b) the drug has important value or status in saving lives, curing diseases or significantly delaying the progress of diseases, including the diagnosis of the above diseases. If it is not used, the clinical diagnosis and treatment work and patients' health outcomes will be affected Significant. Drugs conforming to these two points are clinically important drugs, otherwise they can be regarded as clinically unimportant drugs.

Taking chemical drugs as an example, based on the convenience of switching between drugs and the potential impact on treatment, alternative drugs can be divided into five levels:

- Other drugs with the same general name, indications, usage and dosage of the drug and proved to be equivalent in treatment;

- Other drugs which are only the same as the active ingredients, usage, dosage and indications of the drug, but different in dosage form and base, but have been proved to be equivalent in treatment;

- Other drugs with the same active ingredients and indications, but different dosage forms, usage, base, etc;

- Other drugs that are only considered to be able to treat the disease in the "guidelines for diagnosis and treatment" or expert consensus but have not been approved for the indication;
- Only other drugs with the same or similar pharmacological classification and mechanism of action but lack of clinical evidence.

In this grading system, the lower the grade, the worse the convenience of substitution and the greater the potential impact on treatment. Detailed description of the selection criteria of alternative drugs is not only conducive to the classification of drugs in shortage, but also can meet the needs of medical institutions in dealing with drugs in shortage.

The expected recovery time of shortage drugs needs to be focused. Through the research on the duration of drug shortages in domestic medical institutions, Chinese scholars found that the duration of drug shortages from the emergence of shortage to the recovery of supply presents a trend of polarization, and most of the duration of drug shortages is concentrated in the time range of $<3$ months and $>6$ months [18-20]. The duration of drug shortages is closely related to the degree of impact. When there is a temporary shortage of drugs, the stock of drugs in medical institutions or patients may play a certain role in alleviating, and the longer the duration of drug shortages, it may produce a series of adverse consequences. In addition, there is also a correlation between the expected supply recovery time and mitigation measures. For example, if a drug cannot be supplied normally for only one month in a row, it only needs to help the drug manufacturer to resume production as soon as possible, and no other mitigation measures are needed. Therefore, by referring to the existing classification standards of shortage drugs and domestic literature survey, the expected supply recovery time is divided into three levels: long-term shortage (shortage duration $>6$ months), medium-term shortage (shortage duration 3- 6 months) and short-term shortage (shortage duration $<3$ months).

A domestic study on a medical institution in China shows that when choosing alternative drugs, the proportion of drugs with the same specification from different manufacturers is the highest (51.4\%), which indicates that when drugs are in shortage, alternative drugs with the same common name are used in most cases (that is, other drugs with the same common name, indication, usage and dosage of the drugs in shortage, and have been proved to be equivalent) [21]. However, the industrial structure of China's pharmaceutical market is unreasonable. Once the pharmaceutical manufacturers with large market share interrupt production, other 
pharmaceutical manufacturers may not be able to supplement the market demand in a short time. In addition, the fewer the manufacturers, the more serious the drug shortages [22]. Zhou he's research on the shortage of drugs in Liaoning Province from January to June 2018 shows that the number of manufacturers with shortage of drugs is $\leq 5$ [23]. Therefore, when there is a shortage of drugs substituted by the same generic name, we should not only evaluate the expected recovery time of the shortage drugs, but also consider the national market share of the shortage drugs. The higher the national market share of the shortage drugs, the higher the risk of drug shortages and the greater the impact. Based on this, the national market share of pharmaceutical manufacturers is divided into three levels: high (national market share between $50 \%$ and $100 \%$ ), medium (national market share between $25 \%$ and $50 \%$ ), and low (national market share less than 25\%).

\section{Classified management and application of drug shortages}

To sum up, in order to avoid the serious impact of drug shortages on public health, the existing management and control measures should be sorted out and optimized, the lack of measures should be supplemented and improved, and the efficiency of existing mitigation strategies should be improved. The classification system should be established with the clinical importance, the availability of alternative drugs and the impact of shortage as the main indicators. In practice, the responsibilities and obligations of stakeholders in the drug supply chain are stipulated by combining the causes of China's shortage and classification results.

Globally, problems in the supply of APIs are a common cause of drug shortages. According to a Finnish study, in 30 interviews on the causes of drug shortages, 21 times were mentioned that the supply of APIs is hindered [24]. Although China is a big producer of API, the shortage of API in China often occurs. For example, in the first three quarters of 2018, the number of drugs that could not be normally supplied due to insufficient production capacity of production enterprises in Liaoning Province was as high as 16 [25-27]. Xu retrospectively analyzed the drug shortages information of a hospital from 2016 to 2018 and found that the shortage of raw materials was the primary cause of drug shortages (accounting for $37.4 \%$ ) [21]. Some pharmaceutical manufacturing enterprises do not produce API or only rely on a few API enterprises, and their production activities are easily affected by the supply of API. However, China has not formulated specific control measures on how to manage the drug shortages caused by the difficulty in obtaining API. Therefore, the corresponding strategies can be formulated on the basis of the classification system. For drug manufacturers, different API management plans can be formulated according to the classification of drugs in short supply. Risk management plan is helpful to prevent and alleviate many aspects of drug shortages [28]. Due to the different clinical importance, substitutability and impact of shortage of drugs produced by drug manufacturers, the requirements of API risk management plan are also different. The API risk management plan of manufacturers of clinically important drugs in short supply includes: (1) having at least two API suppliers; (2) constantly searching for better API suppliers to realize optimization; (3) regularly inspecting the production status of API suppliers to prevent supply interruption; (4) selecting reliable third-party organizations to manage the supply of API, etc. The content of API risk management plan of manufacturers of clinically unimportant drugs in short supply includes: (1) timely understanding of API price changes to prevent monopoly; (2) maintaining appropriate API inventory, etc. It is an effective measure for drug manufacturers to take appropriate risk management through the classification results of shortage drugs, which can largely prevent and alleviate the drug shortages caused by the problem of API. On the regulatory side, in order to cooperate with and ensure the formulation and implementation of risk management plan for drug manufacturing enterprises, regulatory agencies should issue corresponding policies to reflect the regulatory flexibility of ensuring the production and supply of drugs in short supply. For example, when pharmaceutical manufacturers for important clinical uses apply for increasing API suppliers in order to prevent shortage of APIs, the regulatory authorities should encourage this practice by taking measures such as speeding up approval and ensure that the corresponding regulatory authorities in the whole registration process can give recognition. For the manufacturers of clinically unimportant drugs in short supply, the regulatory authorities should establish daily supervision on the stock and price of APIs of the manufacturers of drugs in short supply, so as to prevent drug shortages due to problems in the supply of APIs.

\section{Conclusion}

At present, the prevention and mitigation strategies for the shortage of drugs are relatively perfect, but the utilization and operability in the practical application process are not high. Establish the classification management system of shortage drugs, divide the 
shortage drugs according to the attributes of shortage drugs, the availability of alternative drugs and other indicators, and determine the follow-up regulatory actions based on the classification results, so as to improve the efficiency of relevant measures and policies, make the existing control methods more efficient and flexible, and reduce the serious impact of shortage drugs on the public health system. Through the accurate classification of the shortage of drugs so as to establish the risk-assessment and risk-management strategies for the mitigation of the shortage effects in different healthcare settings. In addition, regulatory agencies and other stakeholders can carry out reasonable coordination and communication according to the classification of drugs in shortage.

Based on the analysis of the current situation and application of classified management of shortage drugs in China, this study discussed the indicators and application of classified management, which has certain limitations. In terms of the availability of alternative drugs, the influence of the price factor of alternative drugs is not discussed. However, we believe that drug shortages are a global topic, and all regulatory agencies are facing challenges, the system can be further optimized and upgraded to enrich its application in different environments.

\section{Acknowledgments}

The author would like to thank all the experts and scholars who have made great efforts in this paper.

\section{Funding}

No funding was received to assist with the preparation of this manuscript.

\section{Conflicts of Interest}

The authors have no financial or proprietary interests in any material discussed in this article.

\section{Availability of Data and Material}

Not applicable.

\section{Author Contributions}

- Bing Fang: Data extraction and analysis and wrote manuscript.

- Zhen Chen: Conceived of or designed study, drafting the work or revising it critically for important intellectual content.
- Xiaohe Li: Research and refine the literature. Hui Chen: Analyzed data.

- Jianhong Yang: Performed research and designed study.

\section{Bibliography}

1. Videau M., et al. "Drug shortages in Canada: Data for 20162017 and perspectives on the problem". Annales Pharmaceutiques Françaises 77.3 (2019): 205-211.

2. Ventola CL. "The drug shortage crisis in the United States: causes, impact, and management strategies". P\&T 36.11 (2011): 740-757.

3. Dal Moro F. "BCG shortage in Europe". Preventive Medicine 57.2 (2013): 146.

4. Romano S., et al. "Time-trend analysis of medicine sales and shortages during COVID-19 outbreak: Data from community pharmacies". Research in Social and Administrative Pharmacy 17.1 (2021): 1876-1881.

5. Badreldin HA and Atallah B. "Global drug shortages due to COVID-19: Impact on patient care and mitigation strategies". Research in Social and Administrative Pharmacy 17.1 (2021): 1946-1949.

6. National Health Commission of the People's Republic of China. "Guidelines for classification and substitution of drug shortages" (2019).

7. Hu A., et al. "Investigation and analysis of the reasons for drug shortage from 26 medical institutions in China". China Pharmacy 28.27 (2017): 3754-3758.

8. Health Commission of Shandong Province. "Work plan of drug shortages monitoring in Shandong Province". 21 June (2016).

9. Li P., et al. "Cause analysis and prevention of drug shortages in medical institutions". Journal of Pharmaceutical Practice 33.2 (2015): 179-182.

10. Chen H., et al. "Analysis of the investigation and responses to drug shortage in 63 medical institutions of 18 provinces and cities". Pharmaceutical Care and Research 16.5 (2016): 331334. 
11. Sheng Y., et al. "Study on the policy of shortage drugs supply in China" (2017): 57-60.

12. Parenteral Drug Association (PDA). "Risk-Based Approach for Prevention and Management of Drug Shortages". Technical Report No. 68. Parenteral Drug Association, Inc (2014).

13. Musazzi UM., et al. "New regulatory strategies to manage medicines shortages in Europe". International Journal of Pharmaceutics 579 (2020): 119171.

14. U.S. Food and Drug Administration. "Drug Shortage Manual of Policies and Procedures (MaPP)". 30 November (2018).

15. European Medicines Agency. "Criteria for classification of critical medicinal products for human and veterinary use". 17 March (2016).

16. Shang C. "Study on the construction of national essential drug shortages risk evaluation index system". M.Sc. Thesis. Beijing University of Chinese Medicine (2017).

17. Hantel A., et al. "Prevalence and Severity of Rationing During Drug Shortages: A National Survey of Health System Pharmacists". JAMA Internal Medicine 179.5 (2019): 710-711.

18. Ma L., et al. "Statistical and analysis of drug shortage in 52 sentinel hospitals in Hubei Province". China Pharmacist 23.5 (2020): 926-930.

19. Chen Y., et al. "Current situation and countermeasure of drug shortage in a grade-III class A hospital". China Pharmaceuticals 28.17 (2019): 76-78.

20. Yan J., et al. "Investigation and reason analysis of drug shortage in 78 medical institutions from Sichuan Province". China Pharmacy 30.8 (2019): 1014-1018.

21. Xu X., et al. "Analysis of drug shortage characteristics and countermeasures in a hospital". China Pharmacist 23.2 (2020): 326329.

22. Cai W., et al. "On the current situation of drug shortage in Shaanxi Province based on the provincial monitoring platform". Chinese Pharmaceutical Affairs 31.12 (2017): 1420-1425.

23. Zhou H., et al. "Drug shortage warning in Liaoning Province from January to June 2018". Chinese Journal of Pharmacovigi- lance 17 (2020): 44-50.

24. Heiskanen K., et al. "The reasons behind medicine shortages from the perspective of pharmaceutical companies and pharmaceutical wholesalers in Finland". PLoS One 12.6 (2017): e0179479.

25. Liaoning Provincial Drug and Medical Consumables Centralized Purchasing Office. Notice on Issuing No.1 early warning forecast of drug shortage in Liaoning Province in 2018.

26. Liaoning Provincial Drug and Medical Consumables Centralized Purchasing Office. Notice on Issuing No.2 early warning forecast of drug shortage in Liaoning Province in 2018.

27. Liaoning Provincial Drug and Medical Consumables Centralized Purchasing Office. Notice on Issuing No.3 early warning forecast of drug shortage in Liaoning Province in 2018.

28. Miljković N., et al. "Prospective Risk Assessment of Medicine Shortages in Europe and Israel: Findings and Implications". Frontiers in Pharmacology 11 (2020): 357.

\section{Assets from publication with us}

- Prompt Acknowledgement after receiving the article

- Thorough Double blinded peer review

- Rapid Publication

- Issue of Publication Certificate

- High visibility of your Published work

Website: www.actascientific.com/

Submit Article: www.actascientific.com/submission.php Email us: editor@actascientific.com

Contact us: +919182824667 